\title{
Disposition Start Date
}

National Cancer Institute

\section{Source}

National Cancer Institute. Disposition Start Date. NCI Thesaurus. Code C83125.

The date on which the disposition event begins. 